\title{
21. THE PROBLEM OF ELABORATION AND CLASSIFICATION OF OBSERVATIONAL MATERIAL FOR ONE-APPARITION COMETS
}

\author{
M. BIELICKI \\ Astronomical Observatory, Warsaw University, Warsaw, Poland
}

\begin{abstract}
A new, purely objective criterion is developed for the rejection of observations with nonaccidental errors. A mathematically rigorous procedure is devised for the determination of the weights of individual observations.
\end{abstract}

For the investigation of orbits of one-apparition comets from a statistical point of view it is necessary to discuss all the comets in the same manner, using the same astronomical constants and the same methods for reducing the observations and utilizing the observational material.

The first problem will then be to introduce systematic corrections to the observations: corrections due to differences between the star catalogues (including the constants of precession, nutation and aberration, and the proper motions of the stars) and between methods of reduction, time scales, etc.

The other important problem in the homogenization of the observations is the quite objective, purely mathematical question as to the best use of the observational material. It is known that observations of the celestial coordinates of a comet at specified moments of time are just the particular values of the observed variables arising from the general functional dependence between the elements of the orbit and the position in the sky at these times. But the observational data of position and time are influenced by errors of various types and are of different weights. We may simplify the problem arbitrarily by putting it in a fictitious form in which we consider the observed times to be completely free from error but introduce the errors that necessarily exist in the times into further errors in the positions. We consider therefore the positions as particular values of a random variable and the mean square error of the positions as the dispersion of this random variable.

Summing up, we take into consideration two properties of an observation: its weight and its correctness of performance and elaboration. These two problems have been investigated analytically and numerically and solved with the help of statistical methods. We present here a brief account of these investigations and their results.

First let us consider briefly the classification of observations from the point of view of their doubtfulness, i.e., the conventional classification as a set of observations with accidental errors only. This implies that the observations should have been obtained and reduced correctly within the possibilities of the instruments used and the methods of observation. There are many methods used for this classification mainly the ones for rejecting observations with nonaccidental errors - from the naïve guess or primitive criterion such as $3 \sigma$ to procedures that are very complicated both analytically and numerically and well-nigh impossible to apply. 
Among these methods one draws particular attention because it is fairly objective and easy to handle numerically. This method, based on Chauvenet's (1891) reasoning, derives from a simple and sound principle: the probability that an error will exceed a given magnitude $\sigma \theta$ is $1-P(\theta)$, where $\sigma$ denotes the dispersion, $\theta$ the limiting coefficient, and we have

$$
P(\theta)=\frac{2}{\pi} \int_{0}^{2-1 / 2 \theta} e^{-t^{2}} \mathrm{~d} t
$$

When $N$ observations are made the probable number of those surpassing $\sigma \theta$ is $N[1-P(\theta)]$. If $N[1-P(\theta)]=1 / 2$, then

$$
P(\theta)=(2 N-1) / 2 N .
$$

Let $\varepsilon$ be an error such that if $|\varepsilon|>\sigma \theta$, the corresponding observation is to be rejected on the ground that it will have a greater probability against it than for it. The numerical application is thus based on the consecutive formulae

$$
\begin{aligned}
P\left(\theta_{\mathrm{Cr} 1 / 2}\right) & =1-\frac{1}{2 N} \\
P\left(\theta_{\mathrm{Cr} 1 / 2}\right) & =\frac{2}{\pi} \int_{0}^{2-1 / 2 \theta_{\mathrm{Cr} 1 / 2}} e^{-t^{2} \mathrm{~d} t} \\
\sigma_{\mathrm{Cr} 1 / 2} & =\sigma \theta_{\mathrm{Cr} 1 / 2},
\end{aligned}
$$

where we define this criterion as $\mathrm{Cr} 1 / 2$.

The coefficient $\theta_{\mathrm{Cr} 1 / 2}$ is a function only of $N$ and can be tabulated. The reasoning is based on the fact that the probable number of residuals (a residual has to replace the deviation of a measurement from the truth) larger than the limit $\sigma_{\mathrm{Cr} 1 / 2}$ is smaller than $1 / 2$, which means that measurements with such residuals should not exist: if any do, they apparently suffer from an abnormal source of error and should be rejected. The process should of course be repeated to the limit, where none of the measurements retained has a residual exceeding the corresponding value $\sigma_{\mathrm{Cr} 1 / 2}$.

In principle, the criterion $\mathrm{Cr} 1 / 2$ seems to be reasonably correct, and what is more important, it is quite objective. It has, however, a drawback resulting from the fact that the factor $\sigma$ in the calculation of the limit $\sigma_{\mathrm{Cr} 1 / 2}$ is a random variable as well. Therefore in actual applications this factor leads to uncertainty in classifying measurements into those with accidental and those with nonaccidental errors.

The random variable $\sigma$ has a distribution in which the probable error can be estimated. This error is equal to

$$
\frac{0.4769363 \ldots}{\sqrt{N}} \sigma
$$

hence the condition

$$
{ }_{\text {theor }} \sigma\left(1-\frac{0.47 \ldots}{\sqrt{\bar{N}}}\right)<_{\text {real }} \sigma<_{\text {theor }} \sigma\left(1+\frac{0.47 \ldots}{\sqrt{\bar{N}}}\right)
$$


is realized with probability $1 / 2$, and so is the condition

$$
{ }_{\text {real }} \sigma\left(1+\frac{0.47 \ldots}{\sqrt{N}}\right)^{-1}<\text { theor } \sigma<_{\text {real }} \sigma\left(1-\frac{0.47 \ldots}{\sqrt{N}}\right)^{-1} \text {. }
$$

In our case the criterion $\mathrm{Cr} 1 / 2$ is based on the mean value, i.e., on the theoretically accepted theor $\sigma={ }_{\text {real }} \sigma$. We obtain the limiting condition for theor $\sigma$ when we accept that this criterion should work when the probability of existence of a class of nonaccidental errors in the range from a vanishing state to the theoretical mean state attains a value of not more than $1 / 2$. This is the condition when the probability of existence of ${ }_{\text {theor }} \sigma$ reaches the value $1 / 2$ in an increasing direction from the mean value.

According to the above reasoning, taking into account the uncertainty of the expression theor $\sigma$, we accept as our basis the formula

$$
\sigma_{\mathrm{Cr} 1 / 2,1 / 2}=\sigma\left(1-\frac{0.47 \ldots}{\sqrt{N}}\right)^{-1} \theta_{\mathrm{Cr} 1 / 2},
$$

where $\mathrm{Cr} 1 / 2,1 / 2$ represents the new criterion and $\sigma_{\mathrm{Cr} 1 / 2,1 / 2}$ gives us the limiting residual for the rejection of measurements with nonaccidental errors. This new criterion takes into consideration the fact that the number of measurements with residuals larger than the limit should be less than $1 / 2$, with the probability of existence of these measurements also smaller than $1 / 2$ because of the uncertainty of the observational mean square error. It is easy to see that the criterion $\mathrm{Cr} 1 / 2,1 / 2$ is more liberal than the $\mathrm{Cr} 1 / 2$ criterion in classifying the observations as correct, i.e., with accidental errors only. This feature is particularly important when the number of measurements is not too large. On the other hand, the number of measurements cannot be too small, or the statistical methods used for developing the criterion would not be applicable. In practice the minimum value of $N$ is about 20 , the accuracy of the determination of $\sigma_{\mathrm{Cr} 1 / 2,1 / 2}$ being about $10 \%$. In actual examples we have found that application of this criterion causes the elimination of about 10 to $15 \%$ of the observations. Perhaps 60 to $70 \%$ of the observations eliminated can be corrected and recovered; there remains about 3 to $5 \%$ of the whole that is completely lost. Owing to the increasing use of automation in the measurement and reduction of observations we lose a smaller percentage of modern observations. The criteria $\mathrm{Cr} 1 / 2$ and $\mathrm{Cr} 1 / 2,1 / 2$ can be included in computer programmes using tables or suitable functional models. It must be remembered that the necessary condition for application of this criterion is that the observations have equal weights. To investigate the effects of the criteria in sets of observations a large number of numerical tests have been devised and performed on digital computers. The results of these tests and investigations lead us to accept the above criterion $\mathrm{Cr} 1 / 2,1 / 2$ as correct and applicable in the classification of observational material.

Let us take now into consideration the other property of the observations - their different weights. Attention should be drawn to the fact that for the homogenization of the observations it is necessary to consider weights, both when solving the observational equations by the method of least squares and when applying the criterion of 
rejection for observations with nonaccidental errors. When the weights have been determined numerical application is easy.

The weights should be determined in a purely objective way and they must in principle result from the same observational material. There are many methods for giving weights to observations. One can quote from Plummer (1939): "In any case the decision should be based on the intrinsic circumstances which affect the observation: the fact that it may give a result not agreeing with expectation or with the results of other observers, is a most dangerous guide in assessing its value." Indeed, all methods based on this type of expectation are applied incorrectly.

Only the mathematical determination of the local mean error at different points in a series of observations enables us to obtain local weights and hence the whole set of weights for the series. The determination of the local mean square error (i.e., the local dispersion of the observation function) can be carried out fairly easily under certain suppositions. One way of doing it is to consider a subinterval surrounding a point in the series and containing a sufficient number (which cannot be too small) of observations, and to represent this subinterval with some particular function according, say, to the method of least squares. The next step is to determine the dispersion, i.e., the mean square error of a single observation. We suppose that all observations have the same accuracy in this subinterval - it cannot be too long and that they have only accidental errors. It is advisable to take into consideration only observations that are quite certainly reliable, i.e., to base the result on a conventional 'nucleus of accuracy' of observations, defined identically in all the subintervals investigated. This can be achieved if we apply a rather sharp criterion for the local rejection of doubtful observations, separately in each subinterval. It is known from practice that the Chauvenet criterion of $\mathrm{Cr} 1 / 2$ is sufficient for this. This is permissible, since in the determination of sets of weights it is not the values of the mean errors themselves, but the ratios of these errors, that are important. Repetition of this process at different points of the observation interval gives us local mean errors of the 'nucleus of accuracy' type at these points. These local mean errors correspond to definite values of the time and hence they can be interpolated and extrapolated for all points in the series of observations. By choosing a conventional mean square error for the unit of weight we may determine the whole set of observational weights.

The basic problem is the form of the function representing the residuals in the subintervals. The set of residuals depends on this form, and hence the elimination process of doubtful measurements does as well. So does the mean square error of the observations in each subinterval, and hence the weights of the observations.

Two particular cases can be quoted here: the a posteriori type of mean error, resulting from the application of the same functional form as for solving the whole problem; and the a priori type of mean error, demonstrating the observational inaccuracy of a single observation. Both of these types of mean error are of great importance in assigning sets of weights, which therefore can be of different types also.

Accepting as the basis of the solution of a problem the determination of the most probable orbit according to Newtonian gravitation only, i.e., using a posteriori mean 
errors, we shall consider the nongravitational effects as additional influences augmenting the accidental errors of the observations. Then the elements of the orbit will be the best according to Newtonian gravitation and suitable for the detectability of nongravitational effects - their existence and influence on the residuals.

On the other hand, when we use a priori mean errors, the orbit will be the best mean result for both pure Newtonian gravitation and various nongravitational effects. Such an orbit will be particularly suitable for the definitive analysis and determination of such nongravitational effects and the Newtonian orbit of the comet.

Numerical mean errors of the $a$ posteriori type, i.e., after the application of the Newtonian mathematical model, can be obtained immediately. The mean errors of the $a$ priori type must be determined in another way: there does exist a purely mathematical manner for determining them, utilizing variance properties of ordinary and divided differences; see Bielicki $(1958,1967)$. There are many interesting details of application which will not be mentioned here.

It is easy to see that the two problems - the calculation of a set of weights and the elimination of observations with abnormal sources of errors - have some common points, and therefore they are numerically solved together as follows: First we eliminate the observations that are obviously wrong (e.g., the preliminary residuals are more than $50^{\prime \prime}$ ). Then, by means of the subinterval procedure, we determine the local mean square 'nucleus of accuracy' errors (either $a$ priori or $a$ posteriori or some other type) for separate points on the observational interval. Next we find mean errors of the adopted type for particular observations using methods of smoothing, interpolation, and extrapolation, and hence we establish the weights. Since the local mean error depends on the set of residuals, and this set depends on the weights of the observations, the process of weight determination must be an iterative one. It is also connected with the elimination process. The procedure is rapidly convergent, however, and because the weights are not required with very great accuracy (e.g., to about $10 \%$ only), two, three or four approximations already give a good result. After obtaining the final weights we apply them to the observations and then subject the whole observational material to the new classification criterion $(\mathrm{Cr} 1 / 2,1 / 2)$, which eliminates almost all the observations affected by some abnormal source of error.

The problem of weighting and the elimination of doubtful observations has been solved for the case where the total number of observations is more than 20 . When this number is less there are other, simplified methods for application that make use of less information.

We have in general two or more possibilities for the solution, depending on which type of weights we assign to the observations. Each of these possibilities has its importance for further investigation of the motion of the comet.

In connection with the above reasoning about weights, elimination processes, etc., we have performed a large number of investigations and tests on computers. As an example we considered 83 observations of comet 1953 I. A series of experiments was carried out in which we considered different divisions into subintervals, different 
methods and criteria for rejecting doubtful observations, different mathematical models for representing the residuals, etc. Finally we investigated the iterative process for determining mean square errors of the $a$ priori and $a$ posteriori types and the sets of weights that follow from these errors. Figure 1 shows that the a priori mean error increases rather suddenly from $1^{\prime \prime}$ to $3^{\prime \prime}$ about a hundred days before the comet passes perihelion; then it increases more slowly to $4^{\prime \prime}$ at the end of the observational interval. The sudden change of this mean error is accompanied by an increase in the

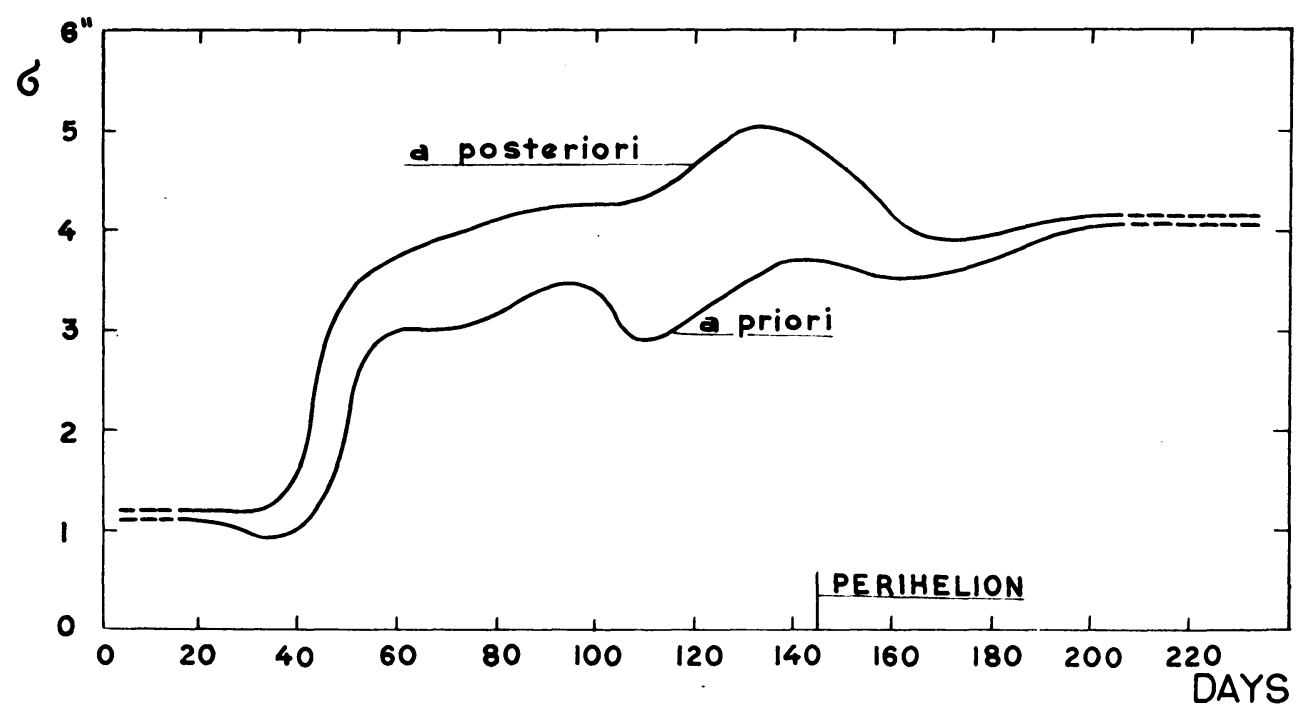

Fig. 1. The local mean errors for comet 1953 I (Harrington).

nongravitational effects, which in turn cause the great increase to $5^{\prime \prime}$ in the a posteriori mean error. The nongravitational influence decreases slowly after perihelion, and both mean errors end up near 4 ". It follows from the changes in the mean errors that the weights of the observations vary considerably during the observational interval, by a factor of 15 for the a priori case and 20 for the $a$ posteriori weights. This has an important effect on determination of the orbit. Further details concerning the application to comet 1953 I are given by Sitarski (1972).

\section{Acknowledgment}

I should like to express my thanks to G. Sitarski for his collaboration, discussion, and assistance with the computing.

\section{References}

Bielicki, M.: 1958, Acta Astron. 8, 131.

Bielicki, M.: 1967, Acta Astron. 17, 409.

Chauvenet, W.: 1891, A Manual of Spherical and Practical Astronomy, 5th ed., Philadelphia, p. 564.

Plummer, H. C.: 1939, Probability and Frequency, London.

Sitarski, G.: 1972, this Symposium, p. 107. 